\title{
Design clues from functional constraints and broadly neutralizing antibodies
}

\author{
Tongqing Zhou ${ }^{1}$, Ling Xu1 ${ }^{1}$, Barna Dey ${ }^{1}$, Ann J Hessell ${ }^{2}$, Shahzad Majeed ${ }^{1}$, \\ Donald Van Ryk ${ }^{3}$, Shi-Hua Xiang ${ }^{4}$, Xinzhen Yang ${ }^{4}$, Mei-Yun Zhang5, \\ Michael B Zwick², James Arthos ${ }^{3}$, Dennis R Burton², Dimiter S Dimitrov ${ }^{5}$, \\ Joseph Sodroski ${ }^{4}$, Richard Wyatt ${ }^{1}$, Gary J Nabel ${ }^{1}$ and Peter D Kwong*1
}

Address: ${ }^{1}$ Vaccine Research Center NIAID, Bethesda, Maryland, USA, ${ }^{2}$ The Scripps Research Institute, La Jolla, California, USA, ${ }^{3}$ Laboratory of Immunoregulation NIAID, Bethesda, Maryland, USA, ${ }^{4}$ Dana-Farber Cancer Institute, Boston, Massachusetts, USA and ${ }^{5}$ Center for Cancer Research and Nanobiology Program NCI, Frederick, Maryland, USA

* Corresponding author

from 2006 International Meeting of The Institute of Human Virology

Baltimore, USA. 17-2I November, 2006

Published: 21 December 2006

Retrovirology 2006, 3(SuppI I):S22 doi:10.1186/1742-4690-3-SI-S22

(c) 2006 Zhou et al; licensee BioMed Central Ltd.

Two strategies have been proposed for the rationale design of vaccine immunogens that elicit broadly neutralizing antibodies against HIV-1. One strategy involves exploiting functional constraints, focusing on regions of the envelope where function requires conservation and exposure. This strategy has lead to the identification of coreceptor-binding-site antibodies as well as antibodies reactive with the site of T-20 binding. One drawback of this strategy, however, is the limited potency of the antibodies thus far identified. A second strategy involves the characterization of rare broadly neutralizing antibodies such as $2 \mathrm{G} 12,2 \mathrm{~F} 5,4 \mathrm{E} 10$ and b12. Unfortunately, strains of virus resistant to these antibodies evolve with relative ease. We used X-ray crystallography to define functional constraints related to binding of the $\mathrm{CD} 4$ receptor and found that the site of initial CD4 contact on gp120 is the epitope for the broadly neutralizing antibody, b12. Functional analysis allowed us to transcend the particulars of b12 binding to focus on the conserved site of initial CD4 contact. The results suggest that a combination of functional analysis and potent antibody characterization may provide design clues necessary to create immunogens capable of eliciting potent broadly neutralizing antibodies. 